\title{
In vivo and in vitro effects of heme oxygenase-1 silencing on the survival of acute myelocytic leukemia-M2 cells
}

\author{
SIXI WEI ${ }^{1-3}$, YATING WANG ${ }^{1,2}$, QIXIANG CHAI ${ }^{1,2}$, QIN FANG $^{4}$, YAMING ZHANG ${ }^{1,2}$ and JISHI WANG ${ }^{1,2}$ \\ ${ }^{1}$ Department of Hematology, The Affiliated Hospital of Guiyang Medical College; \\ ${ }^{2}$ Hematopoietic Stem Cell Transplantation Center of Guizhou Province; \\ Departments of ${ }^{3}$ Clinical Biochemistry and ${ }^{4}$ Pharmacy, The Affiliated Hospital of Guiyang Medical College, \\ Guiyang, Guizhou 550004, P.R. China
}

Received April 8, 2014; Accepted December 11, 2014

DOI: $10.3892 / \mathrm{etm} .2015 .2209$

\begin{abstract}
The aim of this study was to investigate the in vivo and in vitro effects of targeted heme oxygenase-1 (HO-1) silencing on the proliferation and apoptosis of human acute myelocytic leukemia (AML)-M2 cells. Bone marrow mononuclear cells (BMMNCs) were infected by pRNAi-siHO-1-GFP. The viability of the BMMNCs was determined by cell counting kit-8 (CCK-8) assay following daunorubicin (DNR) treatment. The apoptotic rate was detected by flow cytometry. The expression levels of HO-1 and apoptosis-related genes were detected by quantitative polymerase chain reaction (qPCR) and western blot analysis. An AML-M2 xenograft mouse model was established. The tumor formation outcomes and survival were observed. The leukocyte and platelet counts and hemoglobin levels were monitored, and the copy numbers of AML1/ETO fusion gene were detected by qPCR. pRNAi-siHO-1-GFP silenced the expression of HO-1. DNR inhibited cell viability in a time- and dose-dependent manner. The survival rate of the cells was significantly reduced by infection with pRNAi-siHO-1-GFP. HO-1 expression in the BMMNCs infected with pRNAi-siHO-1-GFP was downregulated, whereas caspase-3, -8 and -9 expression was upregulated compared with that in control BMMNCs. Kasumi-1 cells were successfully inoculated into nude mice. The rats inoculated with pRNAi-siHO-1-GFP-transfected Kasumi-1 cells succumbed to tumors more slowly and survived longer than those inoculated with untransfected Kasumi-1 cells. Furthermore, the leukocyte and platelet counts and hemoglobin levels were higher and the copy numbers of AML1/ETO fusion gene were lower in the former group. HO-1 gene silencing may promote the apoptosis of human M2 leukemic cells by inhibiting a caspase-dependent apoptotic pathway. Targeted silencing of HO-1 is able to inhibit
\end{abstract}

Correspondence to: Dr Jishi Wang, Department of Hematology, The Affiliated Hospital of Guiyang Medical College, 28 Guiyi Street, Guiyang, Guizhou 550004, P.R. China

E-mail: jishiwangdoc@126.com

Key words: heme oxygenase-1, proliferation, apoptosis, infiltration the proliferation and infiltration of leukemic cells in nude mice and thus prolong their survival. The findings provide valuable experimental evidence for the molecular targeted therapy of M2 leukemia.

\section{Introduction}

Acute myelocytic leukemia (AML), which results from the malignant cloning of myeloid progenitor cells in the hematopoietic system, is mainly treated by chemotherapy and hematopoietic stem cell transplantation currently. However, the disease-free and overall survival rates remain low due to the toxicity and high recurrence rate of chemotherapy, strict selection of transplant donors and recipients and severe complications $(1,2)$. Therefore, the identification of eligible targets for AML therapy is now of global interest.

Heme oxygenase-1 (HO-1), also known as heat shock protein (HSP) 32, is located on human chromosome $22 \mathrm{q} 12$ while being highly conserved and its expression can be induced by various stimulatory factors (3). HO-1 affects the growth of solid tumors in many aspects, and it is expressed at higher levels in cancer cells, for example, in renal cell carcinoma, squamous cell carcinoma, brain cancer, Kaposi's sarcoma, lung cancer, pancreatic cancer and melanoma, than in normal tissues (4-6). In many types of tumors, HO-1 protects cells from apoptosis, promotes cell proliferation and enhances tumor invasion (7-9). Furthermore, the HO-1 level is associated with the occurrence and development of hematological diseases. When highly expressed, it protects leukemia cells from chemotherapy drugs, reduces apoptosis and enhances drug resistance (11-13). Our group has been studying the association between HO-1 and leukemia. It has been found that the inhibition of HO-1 expression can increase the sensitivity of AML mononuclear cells to chemotherapy (14). In addition, HO-1 has been demonstrated to be involved in the regulation of the survival and apoptosis of chronic myeloid leukemia (CML) cells and to be associated with CML disease progression and drug resistance (15-17). Since HO-1 is significantly highly expressed in patients with leukemia compared with normal subjects, downregulating HO-1 expression may provide new protocols for the treatment of leukemia. Nevertheless, little has been reported about the effects of downregulated HO-1 expression on the proliferation 
and apoptosis of AML cells. In particular, relevant in vivo and in vitro studies remain scarce.

Therefore, HO-1 expression in human AML-M2 bone marrow mononuclear cells (BMMNCs) was silenced through infection using a lentiviral vector with HO-1 small interfering RNA (siRNA). The effect of silenced HO-1 gene expression on the proliferation and apoptosis of mononuclear cells was evaluated in vitro. In addition, an AML1/ETO-positive Kasumi-1 cell-inoculated AML-M2 xenograft mouse model was established to explore the effects of targeted silencing of HO-1 expression by the green fluorescent protein (GFP)-expressing plasmid pRNAi-siHO-1-GFP on the in vivo proliferation and infiltration of leukemic cells. The results should provide experimental evidence for verifying the role of HO-1 regulation in the treatment of AML-M2.

\section{Materials and methods}

Samples. Bone marrow/peripheral blood mononuclear cells from AML-M2 patients and the AML-M2 Kasumi-1 cell line (The Center Laboratory of the Hematopoietic Stem Cell Transplantation Center of Guizhou Province, Guiyang, China) were used as samples.

Testing of HO-1 expression in AML-M2 patients by reverse transcription-polymerase chain reaction (RT-PCR). Twenty peripheral blood samples were collected from AML-M2 patients and another 20 were collected from normal subjects. This study was conducted in accordance with the Declaration of Helsinki and with approval from the Ethics Committee of Guiyang Medical College (Guiyang, China). Written informed consent was obtained from all participants. Mononuclear cells were separated using Ficoll-lymphocyte separation medium (Beyotime Institute of Biotechnology, Nanjing, China). Total RNA was isolated and purified from cells using the RNeasy kit (Qiagen, Hilden, Germany). For RT-PCR analysis, 2,000 ng of RNA was reverse transcribed into cDNA with the Omniscript Reverse Transcription kit (Qiagen) in $20 \mu l$ of reaction volume. Amplification was performed by Veriti ${ }^{\circledR}$ 96-Well Fast Thermal cycler (Applied Biosystems, Foster City, CA, USA). An equal amount of mRNA was loaded and run on a 5\% SDS-PAGE gel. Details of the primers (HO-1 $\left.{ }^{\mathrm{RT}}\right)$ are listed in Table I. Conditions for the RT-PCR reaction were as follows: $40 \mathrm{sec}$ at $53^{\circ} \mathrm{C}$ and $58^{\circ} \mathrm{C}, 6$ min at $94^{\circ} \mathrm{C}$, followed by 30 cycles, each consisting of $40 \mathrm{sec}$ at $94^{\circ} \mathrm{C}$ and $50 \mathrm{sec}$ at $72^{\circ} \mathrm{C}$. The relative expression of HO-1 was demonstrated by the ratio of the gray scale between HO-1 and GAPDH.

Conventional culture of Kasumi-1 cells. The Kasumi-1 cells were maintained in Roswell Park Memorial Institute-1640 (RPMI-1640; Hangzhou Sijiqing Biological Engineering Materials Co., Ltd., Hangzhou, China) culture medium containing 10\% fetal bovine serum (Hangzhou Sijiqing Biological Engineering Materials Co., Ltd.) at $37^{\circ} \mathrm{C}$ in saturated humid air in a $5 \% \mathrm{CO}_{2}$ incubator. The cells were passaged every 3-4 days when the culture medium was also refreshed, and they were inoculated at the density of $1 \times 10^{5} / \mathrm{ml}$.

Preparation and culture of BMMNCs. Ten AML-M2 patients (five males and five females) enrolled in the Hematopoietic

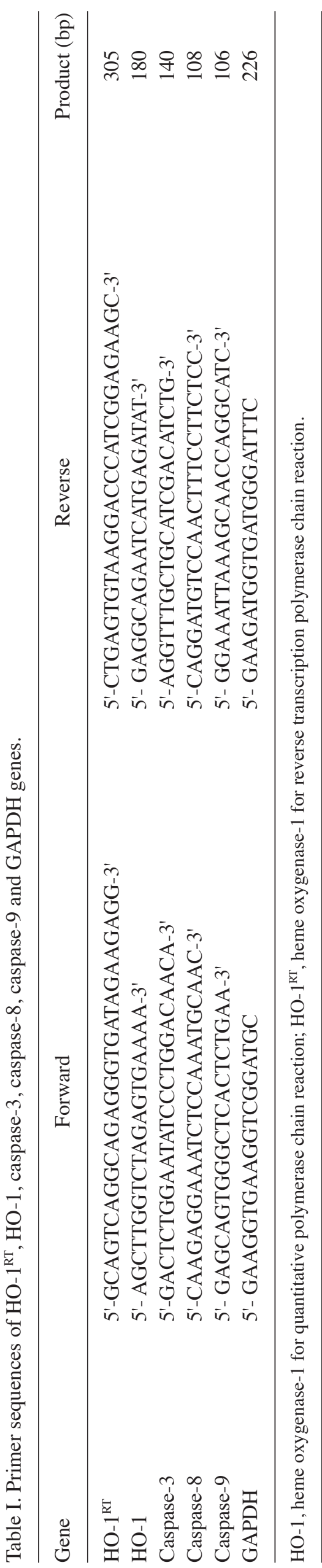


Stem Cell Transplantation Center of Guizhou Province (Guiyang, China) from March 2010 to March 2012 were selected. Under sterile conditions, $2 \mathrm{ml}$ bone marrow blood was drawn, into which was added $2 \mathrm{ml}$ Ficoll-lymphocyte separation medium (relative density: 1.007). After the sample was subjected to horizontal centrifugation at $229 \mathrm{x} \mathrm{g}$ for $10 \mathrm{~min}$, the mononuclear cell layer was collected, washed and precipitated as BMMNCs. The rate of viable cells was $>90 \%$, as indicated by staining with $0.4 \%$ trypan blue. The separated BMMNCs were inoculated in RPMI-1640 culture medium containing $20 \%$ fetal bovine serum, $100 \mu \mathrm{g} / \mathrm{ml}$ penicillin and $100 \mu \mathrm{g} / \mathrm{ml}$ streptomycin, and were cultured and passaged at $37^{\circ} \mathrm{C}$ in saturated humid air in a $5 \% \mathrm{CO}_{2}$ incubator.

Infection of BMMNCs with the recombinant lentivirus pRNAi-siHO-1-GFP. The siRNA sequence for the coding sequence of HO-1 mRNA was designed as follows: 5'-AAG CUUUCUGGUGGCGACAGU-3'. HO-1-targeted siRNA, pRNAi-siHO-1-GFP and pRNAi-GFP were packaged and produced by Biomics Biotechnologies Co., Ltd. (Nantong, China). Kasumi-1 cells and BMMNCs in the logarithmic growth phase were inoculated at the concentration of $1 \times 10^{6}$ cells/well in 6 -well plates following overnight culture and were subsequently transfected with pRNAi-GFP (with/without siHO-1) at multiplicities of infection of 8 in serum-free medium. Polybrene $(5 \mu \mathrm{g} / \mathrm{ml}$; Sigma-Aldrich, St. Louis, MO, USA) was added to improve transfection efficiency as an enhancing reagent. After $10 \mathrm{~h}$, the medium was changed for complete medium and the cells without intervention were used as the blank control group The experimental group was further divided into a normal BMMNC group (BMMNC), a pRNAi-siHO-1-GFP-infected BMMNC group (pRNAi-siHO-1-BMMNC) and a GFP empty vector-infected BMMNC group (pRNAi-GFP-BMMNC). The fluorescence intensity of GFP was observed under a fluorescence microscope (BX51; Olympus Corporation, Tokyo, Japan).

Cell viability assay. Three cell groups in the logarithmic growth phase were inoculated onto 96 -well plates at a density of $4 \times 10^{5} / \mathrm{ml}$. After $12 \mathrm{~h}$ of culture, different concentrations of DNR (Sigma-Aldrich; 0, 2.5, 5, 7.5, 10 and $12.5 \mu \mathrm{g} / \mathrm{ml}$ ) were added to the wells. The inhibitory effects of DNR were determined using the cell counting kit (CCK-8) assay (Beyotime Institute of Biotechnology).

Detection of cell apoptosis. Apoptotic cells were analyzed by flow cytometry with propidium iodide (PI) staining (BD Biosciences, San Jose, CA, USA). In brief, cells of the three groups in the logarithmic growth phase were inoculated onto 6 -well plates at a final density of $1 \times 10^{5} / \mathrm{ml}$. After $12 \mathrm{~h}$ of culture, the cells were treated with different concentrations of DNR $(0$, 5 and $10 \mu \mathrm{g} / \mathrm{ml}$ ) for $48 \mathrm{~h}$. Then, $1 \times 10^{5}$ cells were collected and washed twice with pre-cooled 1X phosphate-buffered saline (PBS). After suspending the cells by adding $500 \mu \mathrm{l} 1 \mathrm{X}$ binding buffer (Solarbio Science \& Technology Co., Ltd., Beijing, China) to each tube, they were further stained by adding $5 \mu \mathrm{l}$ Annexin V-FITC solution (Beyotime Institute of Biotechnology) and $5 \mu 1$ PI solution sequentially. Subsequently, the solution was allowed to react for $15 \mathrm{~min}$ in the dark at room temperature, and cell apoptosis was detected by flow cytometry using the BD
FACSCalibur $^{\mathrm{TM}}$ flow cytometer with BD CellQuest ${ }^{\mathrm{TM}}$ software (BD Biosciences, Franklin Lakes, NJ, USA) within $1 \mathrm{~h}$.

Quantitative PCR ( $q P C R)$. pRNAi-siHO-1-BMMNCs in the logarithmic growth phase were inoculated onto 6-well plates at the final density of $1 \times 10^{6} / \mathrm{ml}$. Various concentrations of DNR $(0,5,7.5,10$ and $12.5 \mu \mathrm{g} / \mathrm{ml})$ were added and the cells were cultured for $48 \mathrm{~h}$. RT-PCR was performed using an RT-PCR thermocycler (Applied Biosystems) and quantified using SYBR $^{\circledR}$ Green PCR Master mix (Applied Biosystems) using $1 \mu l$ cDNA in a final reaction volume of $20 \mu l$. The names, sequences and amplified fragments of the primers (Applied Biosystems) are summarized in Table I. The PCR reactions were cycled 45 times after initial denaturation $\left(94^{\circ} \mathrm{C}\right.$ for $1 \mathrm{~min}$ ) with the following parameters: denaturation at $94^{\circ} \mathrm{C}$ for $10 \mathrm{sec}$; annealing at $58^{\circ} \mathrm{C}$ for $15 \mathrm{sec}$ (caspase-3, caspase- 8 and GAPDH), at $60^{\circ} \mathrm{C}$ for $10 \mathrm{sec}$ (caspase-9), or at $64^{\circ} \mathrm{C}$ for $10 \mathrm{sec}$ (HO-1), and extension at $72^{\circ} \mathrm{C}$ for $15 \mathrm{sec}$. GAPDH was used as the internal control. SDS 2.2.1 software (Applied Biosystems) was used to perform relative quantification of the target genes using the $2-{ }^{\Delta \Delta} \mathrm{Ct}$ method.

Western blotting. BMMNCs and pRNAi-siHO-1-BMMNCs in the logarithmic growth phase were inoculated onto 6 -well plates at a final density of $1 \times 10^{6} / \mathrm{ml}$. Following the addition of $5 \mu \mathrm{g} / \mathrm{ml} \mathrm{DNR}$ for $48 \mathrm{~h}$, the cells were washed in PBS, collected, and then lysed in radioimmunoprecipitation assay buffer $(50 \mathrm{mmol} / 1$ Tris- $\mathrm{HCl} ; 150 \mathrm{mmol} / \mathrm{l}$ $\mathrm{NaCl} ; 0.1 \%$ SDS; $0.5 \%$ Na-deoxycholate and 1\% NP-40) containing proteinase inhibitor cocktail and phosphatase inhibitor cocktail (Roche Applied Science, Indianapolis, IN, USA). The lysate was centrifuged at $10,000 \mathrm{x}$ g at $41^{\circ} \mathrm{C}$ for $10 \mathrm{~min}$. The supernatant $(50-100 \mathrm{mg}$ protein) was fractioned by SDS-PAGE using 10\% gels and was transferred electrophoretically to Hybond-enhanced chemiluminescence membranes (GE Healthcare Life Sciences, Piscataway, NJ, USA). The membrane was blocked with blocking buffer (Li-Cor Biosciences, Lincoln, NE, USA) at room temperature for $1 \mathrm{~h}$ and then incubated with the monoclonal primary antibody [rabbit anti-human HO-1 (1:500), rabbit anti-human caspase-9 (1:500), mouse anti-human caspase-3 (1:500), mouse anti-human caspase-8 (1:500) and mouse anti-human $\beta$-actin (1:1,000); Cell Signaling Technology, Inc., Danvers, MA, USA] at $4^{\circ} \mathrm{C}$ overnight. After being washed with PBS with $0.1 \%$ Tween 20 (PBST), the membrane was incubated with horseradish peroxidase-labeled anti-rabbit IgG (1:1,000; Santa Cruz Biotechnology, Santa Cruz, CA, USA) and anti-mouse IgG (1:1,000; Santa Cruz Biotechnology) for $1 \mathrm{~h}$ at room temperature. The membrane was washed with PBST again and detected by electrochemiluminescence (Beyotime Institute of Biotechnology). By using $\beta$-actin expression as the internal reference, the protein expression levels were calculated using Image J software, V4.0 (National Institutes of Health, Bethesda, MD, USA).

Inoculation of nude mice with AML1/ETO-positive Kasumi-1 cells. Under sterile conditions, 40 nude mice aged 5-6 weeks with weights of 18-22 g were selected (Guiyang Medical College). The numbers of male and female mice were equal. 
A

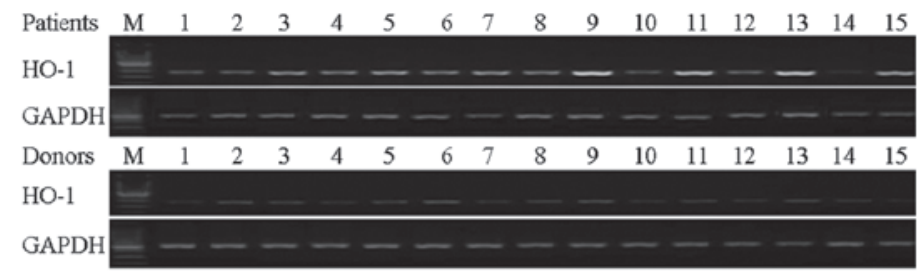

B

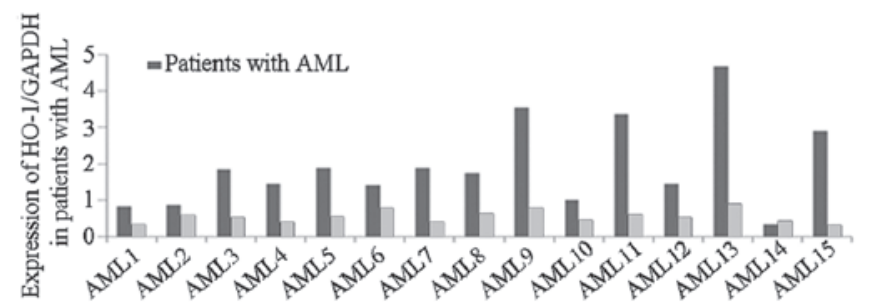

Figure 1. Expression of HO-1 mRNA in AML-M2 patients analyzed by reverse transcription-polymerase chain reaction. (A) HO-1 expression in the peripheral blood mononuclear cells of AML patients was detected by RT-PCR. (B) Relative expression of HO-1 shown in a bar chart using GAPDH as the internal reference. Patients 1-15, AML patients; Donors 1-15, normal subjects; M, marker; HO-1, heme oxygenase-1; AML, acute myelocytic leukemia.

This study was carried out in strict accordance with the recommendations in the Guide for the Care and Use of Laboratory Animals of the National Institutes of Health (The National Academy Of Sciences, Washington D.C, 1996). The animal use protocol was reviewed and approved by the Institutional Animal Care and Use Committee (IACUC) of Guiyang Medical College.

Kasumi-1 cells in the logarithmic growth phase were collected and adjusted to a density of $1,000 \times 10^{4} / \mathrm{ml}$. The mice were divided into four groups ( $n=10$ per group). The blank group was left untreated, the normal saline group was subcutaneously injected with normal saline, the Kasumi group was inoculated with AML1/ETO-positive Kasumi-1 cells $\left(200 \times 10^{4}\right.$ cells/mouse) at the root of the right upper limb, and the pRNAi-siHO-1-K group was inoculated with pRNAi-siHO-1-GFP-transfected Kasumi-1 cells (200x $10^{4}$ cells/mouse) at the root of the right upper limb.

Changes after onset. Following the inoculation of each nude mouse group with Kasumi-1 cells, the body weight changes and the growth of subcutaneous nodules were observed and recorded. The survival times of the mice were evaluated by Kaplan-Meier survival curve.

Routine blood testing. Blood was sampled (0.1-0.2 $\mathrm{ml}$ each time) from the distal end and then the proximal end of the tail vein by making a transverse incision with a disposable sterile scalpel blade. The blood was dropped into a tube containing ethylenediamine tetraacetic acid (EDTA) as an anticoagulant. Routine blood tests were performed to detect the changes of leukocyte and platelet counts as well as the hemoglobin levels.

Detection of fusion gene AML1/ETO expression. Dying mice were killed by cervical dislocation that minimized pain and suffering. The ends of the femur were cut down, and bone marrow was collected. The liver, lung, spleen and kidneys of the mice were detached following the separation of the peritoneal cavity layer-by-layer. Total RNA was extracted from all the aforementioned organs that had been rinsed repeatedly with
A

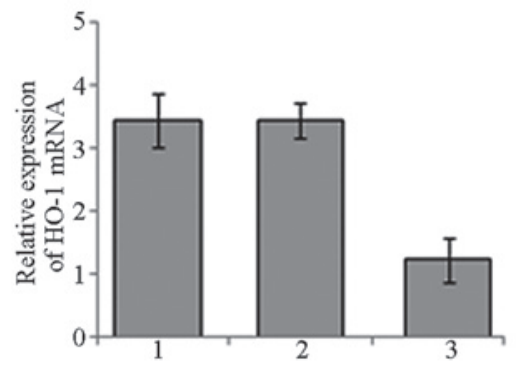

B

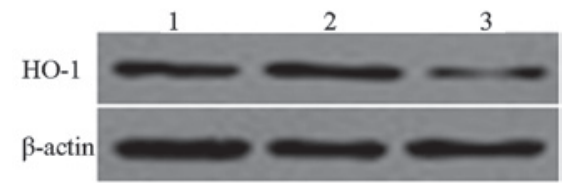

Figure 2. Targeted silencing of HO-1 expression by pRNAi-siHO-1-GFP. (A) After $72 \mathrm{~h}$ of culture, significantly less HO-1 mRNA was expressed in the group infected with lentiviral vector carrying HO-1 siRNA than in the other two groups $(\mathrm{P}<0.05)$. (B) After $72 \mathrm{~h}$ of culture, western blotting results demonstrated that HO-1 protein expression in the pRNAi-siHO-1-BMMNC group was significantly downregulated $(\mathrm{P}<0.05) .1, \mathrm{BMMNC} ; 2$. pRNAi-GFP-BMMNC; 3. pRNAi-siHO-1-BMMNC. HO-1, heme oxygenase-1; GFP, green fluorescent protein; siRNA, small interfering RNA; $\mathrm{BMMNC}$, bone marrow mononuclear cell.

normal saline in order to detect the expression of AML1/ETO by qPCR. The method used TaqMan probes (Applied Biosystems) and an Eppendorf Sequence Detection System (Eppendorf, Hamberg, Germany). The probes and primers sequences used for AML1/ETO qPCR are as described in a Europe Against Cancer Program (18). AML1/ETO, forward: 5'-CACCTACCA CAGAGCCATCAAA-3', reverse: 5'-ATCCACAGGTGAGTC TGGCATT-3' and probe: FAM-AACCTCGAAATCGTACTG AGAAGCACTCCA; ABL, forward: 5'-TGGAGATAACAC TCTAAGCATAACTAAAGGT-3', reverse: 5'-GATGTAGTT GCTTGGGACCCA-3' and probe: FAM-CCATTTTTGGTT TGGGCTTCACACCATT. cDNA templates $(2.5 \mu \mathrm{l})$ were added to each tube as with competitive PCR. Each qPCR assay was performed in a final volume of $25 \mu 1$ under the following conditions: $95^{\circ} \mathrm{C}$ for $5 \mathrm{~min}$, then 40 cycles at $95^{\circ} \mathrm{C}$ for $15 \mathrm{sec}$ and $58^{\circ} \mathrm{C}$ for $1 \mathrm{~min}$. The copy numbers of AML1/ETO were determined according to the standard curve. 
A

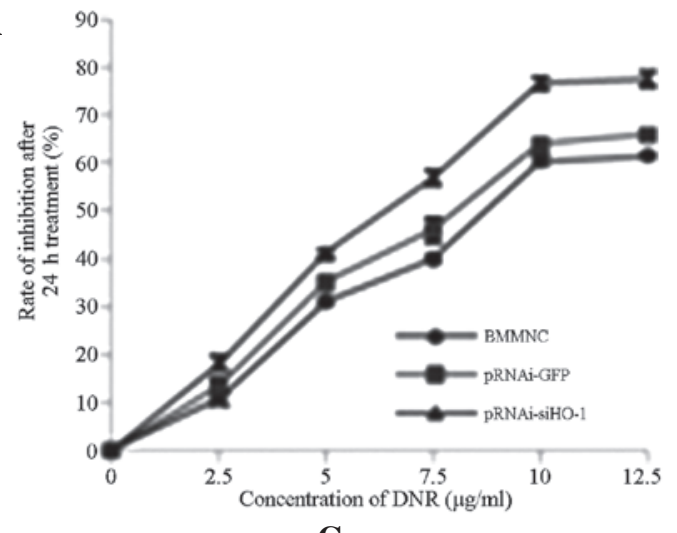

B

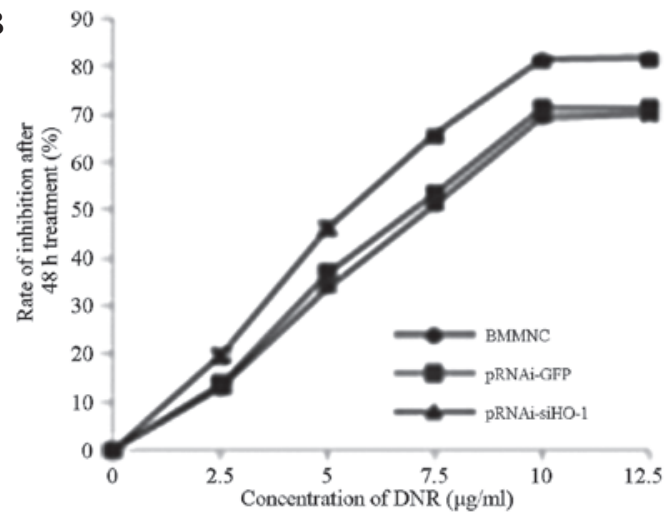

C

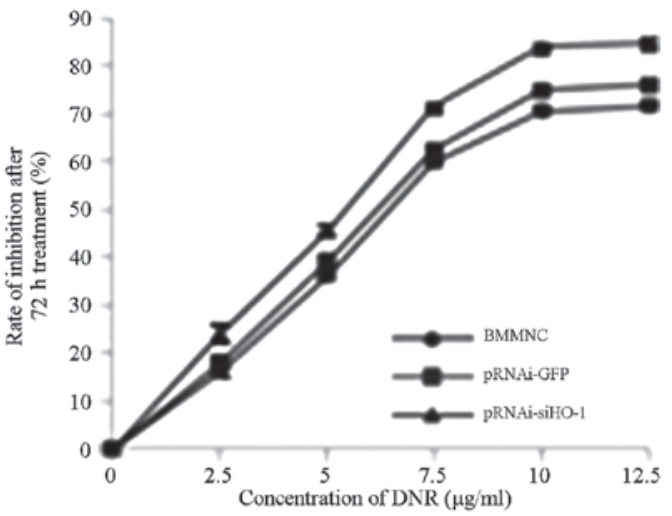

Figure 3. Inhibition of the survival of BMMNCs in various experimental groups by different concentrations of DNR analyzed by the CCK-8 method. Data are expressed as mean \pm standard deviation based on the average of three repeated tests. After being treated with DNR for (A) 24 , (B) 48 and (C) $72 \mathrm{~h}$, respectively, the pRNAi-siHO-1-BMMNC group had significantly higher inhibitory rates than the other two groups did at each drug concentration and at each time interval $(\mathrm{P}<0.05)$. BMMNC, bone marrow mononuclear cell; DNR, daunorubicin; CCK-8, cell counting kit-8; GFP, green fluorescent protein.

Statistical analysis. Data are expressed as mean \pm standard deviation and analysis of variance (ANOVA) was performed with SPSS software, version 13.0 (SPSS Inc., Chicago, IL, USA). $\mathrm{P}<0.05$ was considered as to indicate a statistically significant difference.

\section{Results}

Expression of HO-1 in AML-M2 patients. RT-PCR revealed that $\mathrm{HO}-1$ was expressed at higher levels in the peripheral blood mononuclear cells of AML patients than in normal subjects (Fig. 1).

HO-1 expression in BMMNCs. A lentiviral vector carrying HO-1 siRNA was constructed and used for the targeted silencing of HO-1 expression in BMMNCs. RNA and lysed proteins were extracted from the cells in the experimental groups that had been infected for $72 \mathrm{~h}$. The relative expression levels of HO-1 mRNA and protein were confirmed to be reduced following targeted HO-1 silencing by qPCR and western blotting respectively (Fig. 2).

Effects of targeted HO-1 silencing on survival rate. After 24,48 and $72 \mathrm{~h}$ of treatment with DNR $(0,2.5,5,7.5,10$ and $12.5 \mu \mathrm{g} / \mathrm{ml}$ ) respectively, the effects of HO-1 silencing on DNR-inhibited cell proliferation were determined by the CCK-8 method. As shown in Fig. 3, the survival rates of the BMMNCs correlated with DNR concentration in a time- and dose-dependent manner. After silencing with
pRNAi-siHO-1-GFP, the survival rate of the cells clearly exceeded those of the BMMNC and pRNAi-GFP-BMMNC groups.

Effects of HO-1 silencing on apoptotic rate. The cells treated with 5 and $10 \mu \mathrm{g} / \mathrm{ml}$ DNR for $48 \mathrm{~h}$ were collected and subjected to Annexin V-FITC and PI staining, and the apoptotic rates were measured by flow cytometry (Fig. 4). The apoptotic rate of the BMMNC group increased with rising DNR concentration. The apoptotic rates of the pRNAi-siHO-1-BMMNC group following treatment with 5 and $10 \mu \mathrm{g} / \mathrm{ml}$ DNR were $64.70 \pm 1.99$ and $71.87 \pm 0.96 \%$ respectively, which were significantly higher than those of the BMMNC group (16.24 \pm 0.95 and $36.09 \pm 1.02 \%$, respectively) and the pRNAi-GFP-BMMNC group $(17.90 \pm 1.10$ and $38.73 \pm 1.18 \%$, respectively).

mRNA expression of $\mathrm{HO}-1$ and caspase-3, -8 and -9 . To further explore the role of pRNAi-siHO-1-BMMNC in DNR-induced BMMNC apoptosis, the expression of caspase- $3,-8$ and -9 and HO-1 mRNA was detected by qPCR after $48 \mathrm{~h}$ of treatment with $0,5,7.5,10$ and $12.5 \mu \mathrm{g} /$ $\mathrm{ml}$ DNR. Using GAPDH as the internal reference, relative expression levels were calculated by the $2^{-\Delta \Delta C t}$ method and represented graphically. The expression level of HO-1 in the pRNAi-siHO-1-BMMNC group was lower than that in the BMMNC group (Fig. 5A), while those of apoptosis-related mRNAs increased (Fig. 5B-D). The expression levels of HO-1 and caspase- $3,-8$ and -9 mRNA increased as the concentration of DNR increased. Therefore, HO-1 siRNA 
A
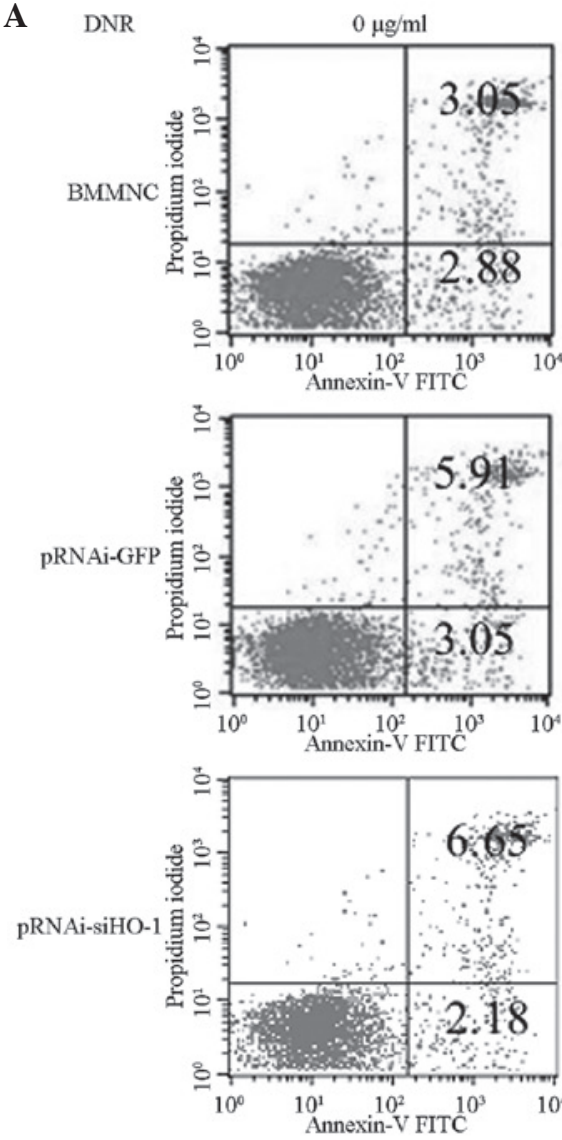
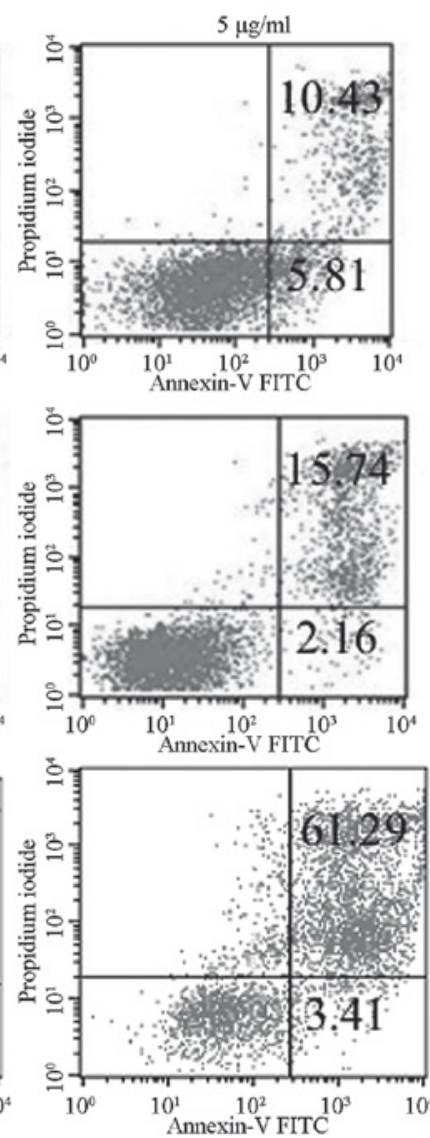
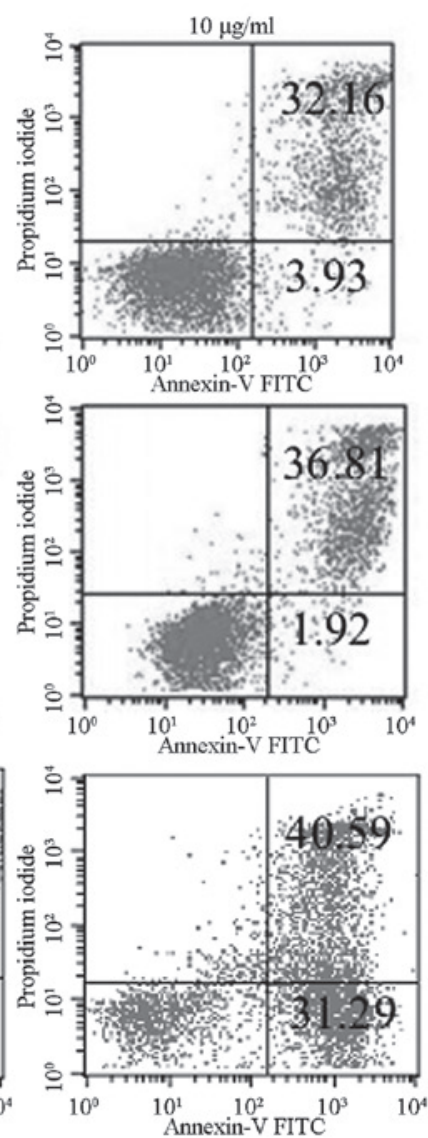

B

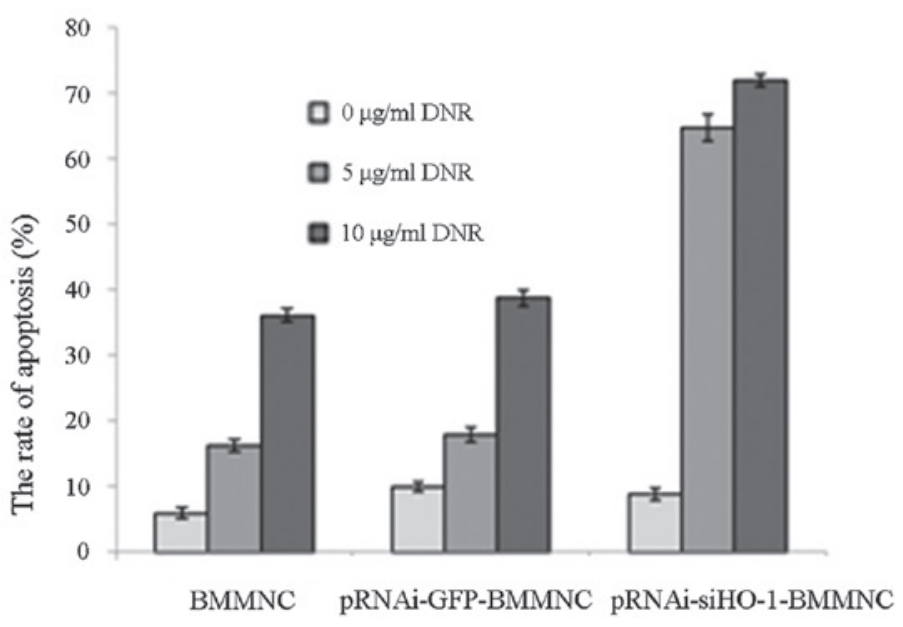

Figure 4. Apoptotic rates of the three cell groups as detected by flow cytometry. (A) DNR induced cell apoptosis in a dose-dependent manner. Following treatment with 5 and $10 \mu \mathrm{g} / \mathrm{ml} \mathrm{DNR}$ for $48 \mathrm{~h}$, the apoptotic rates of the pRNAi-siHO-1-BMMNC group were significantly higher than those of the other two groups $(\mathrm{P}<0.05)$. (B) Apoptotic rates obtained from three repeated experiments (mean \pm standard deviation). DNR, daunorubicin; BMMNC, bone marrow mononuclear cell; GFP, green fluorescent protein; HO-1, hemo oxygenase-1.

may promote BMMNC apoptosis by a caspase activation cascade.

Protein expression of HO-1 and caspase-3, -8 and -9. Lysed proteins were collected from the BMMNC group and the pRNAi-siHO-1-BMMNC group that had been treated with $5 \mu \mathrm{g} / \mathrm{ml} \mathrm{DNR}$. Using $\beta$-actin as the internal reference, the expression of HO-1 and apoptosis-related proteins was detected by western blotting. Following treatment with $5 \mu \mathrm{g} / \mathrm{ml} \mathrm{DNR}$, the expression level of HO-1 in the pRNAi-siHO-1-BMMNC group was lower than that in the BMMNC group, whereas those of the apoptosis-related proteins were higher (Fig. 6). Thus, the HO-1 siRNA-facilitated cell apoptosis was associated with the activation of caspases.

Effects of HO-1 siRNA on the tumor formation outcomes of the established AML-M2 xenograft mouse model. The tumor formation outcomes and nodule volume changes of the four nude mouse groups are presented in Fig. 7A and B. No tumors formed in the blank or normal saline groups. Tumors formed in the Kasumi group on day 6 after inoculation when the nodules were $\sim 0.4 \mathrm{~cm}$ in diameter, and the nodules 
A

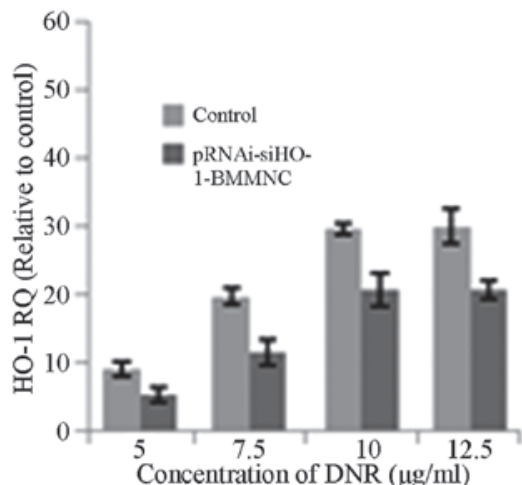

C

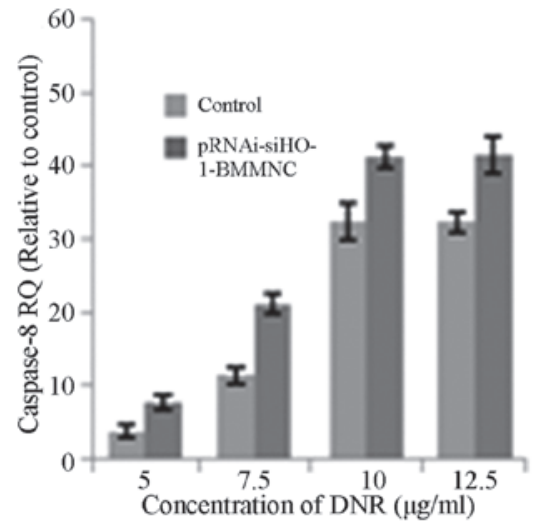

B
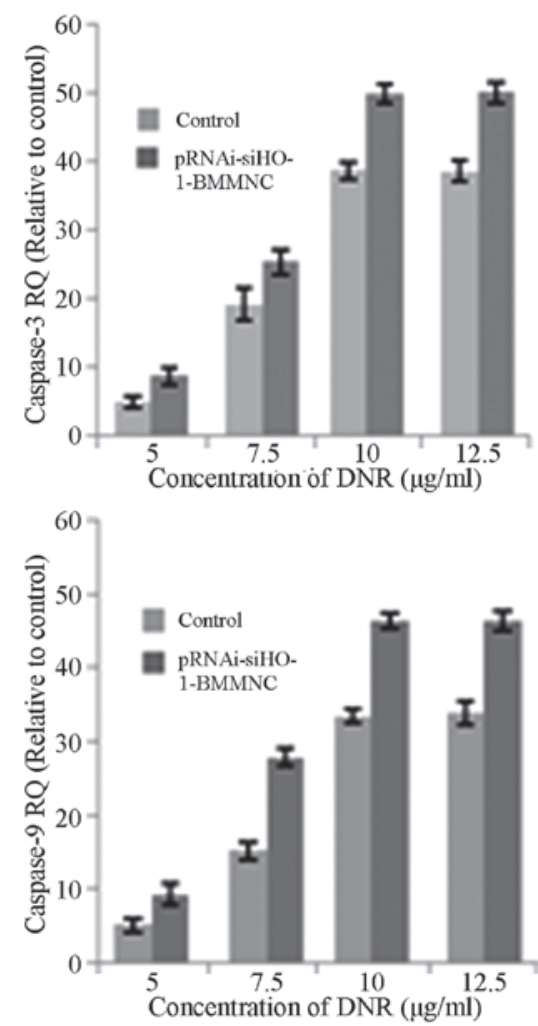

Figure 5. HO-1 and caspase- $3,-8$ and -9 mRNA expression was detected by quantitative polymerase chain reaction. (A) HO-1 mRNA expression changed depending on the dose of DNR. The expression level increased with rising DNR concentration and peaked in the presence of $10 \mu \mathrm{g} / \mathrm{ml} \mathrm{DNR}$. Expression levels of (B) caspase-3, (C) caspase-8 and (D) caspase-9 mRNA in the pRNAi-siHO-1-BMMNC group were higher than those in the BMMNC (control) group at each DNR concentration, the differences in the mRNA expression levels above were statistically significant $(\mathrm{P}<0.05)$. Expression levels increased with rising DNR concentration and peaked in the presence of $10 \mu \mathrm{g} / \mathrm{ml}$ DNR. HO-1, heme oxygenase-1; DNR, daunorubicin; BMMNC, bone marrow mononuclear cell.

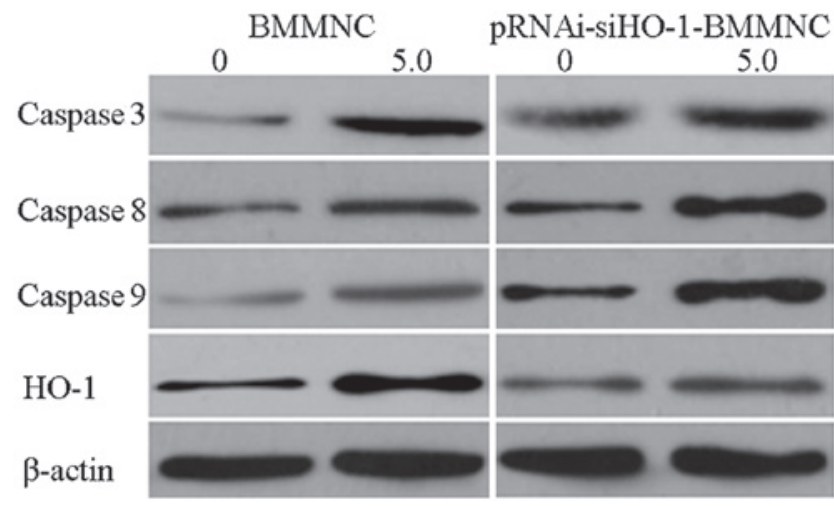

Figure 6. Expression of HO-1 and caspase-3, -8 and -9 proteins. Following treatment with $5 \mu \mathrm{g} / \mathrm{ml}$ DNR, the expression levels of caspase- $3,-8$ and -9 proteins in the pRNAi-siHO-1-BMMNC group were higher than those in the BMMNC group, whereas HO-1 protein expression was silenced. The differences in protein expression were statistically significant $(\mathrm{P}<0.05)$. $\mathrm{HO}-1$, heme oxygenase-1; DNR, daunorubicin; BMMC, bone marrow mononuclear cell.

further grew to $\sim 1.7 \mathrm{~cm}$ on day 15 . The pRNAi-siHO-1-K group developed tumors on day 10 after inoculation, with $\sim 0.3-\mathrm{cm}$-diameter nodules that grew to $\sim 1.2 \mathrm{~cm}$ on day 19 . The body weight changes of the four groups are shown in Fig. 7C. The body weights of the blank and normal saline groups were almost unchanged. By contrast, the Kasumi group underwent a sharp reduction in body weight. The pRNAi-siHO-1-K group underwent a similar body weight reduction to that of the Kasumi group over a longer time period.

Blood samples were drawn from the tail veins of the four nude mouse groups on days 7,14 and 21 after inoculation to determine the changes in the peripheral blood leukocyte and platelet counts as well as the hemoglobin levels. Compared with the blank and normal saline groups, the peripheral blood leukocyte and platelet counts and hemoglobin levels of the Kasumi and pRNAi-siHO-1-K groups were significantly reduced $(\mathrm{P}<0.05)$. The leukocyte counts were $9.25 \pm 0.76 \times 10^{9} / 1$ on day 7 and $7.76 \pm 1.55 \times 10^{9} / 1$ on day 14 in the Kasumi group, and $10.1 \pm 0.42 \times 10^{9} / 1$ on day $7,9.06 \pm 0.72 \times 10^{9} / 1$ on day 14 and $7.22 \pm 0.76 \times 10^{9} / 1$ on day 21 in the pRNAi-siHO-1-K group. The hemoglobin levels were $157.45 \pm 8.8$ and $138.56 \pm 9.82 \mathrm{~g} / \mathrm{l}$ in the Kasumi group on days 7 and 14, and $165.59 \pm 4.92$, $159.06 \pm 6.86$ and $147.22 \pm 10.5 \mathrm{~g} / \mathrm{l}$ in the pRNAi-siHO- $1-\mathrm{K}$ group on days 7, 14 and 21 , respectively. The platelet counts were $935.9 \pm 28.14 \times 10^{9} / 1$ on day 7 and $618.4 \pm 129.89 \times 10^{9} / 1$ on day 14 in the Kasumi group, and $985.8 \pm 16.92 \times 10^{9} / 1$ on day 7 , $798.5 \pm 86.54 \times 10^{9} / 1$ on day 14 and $652.5 \pm 67.26 \times 10^{9} / 1$ on day 21 in the pRNAi-siHO-1-K group. At each time point, the counts of leukocytes and platelets and hemoglobin level of the pRNAi-siHO-1-K group were significantly lower than those of the Kasumi group ( $\mathrm{P}<0.05$; Fig. 7D-F).

In Fig. $7 \mathrm{G}$, the average survival times of the four nude mouse groups are shown to be $41.3 \pm 2.43,40.2 \pm 5.56,18.1 \pm 3.78$ and 34.8 \pm 3.64 days in the blank, normal saline, Kasumi and pRNAi-siHO-1-K groups, respectively. Compared with the 

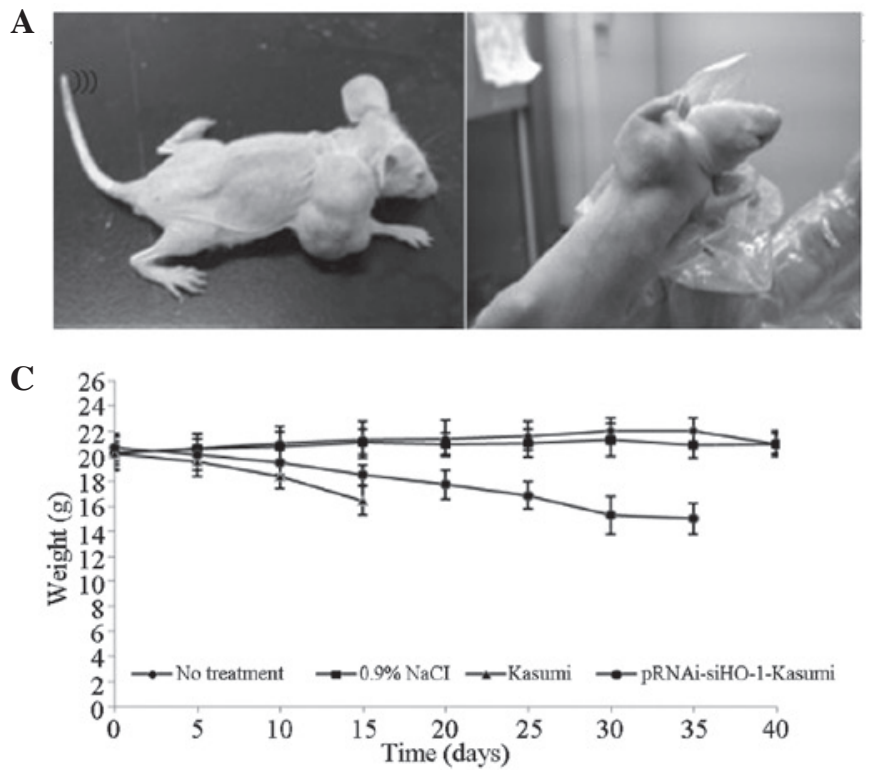

E

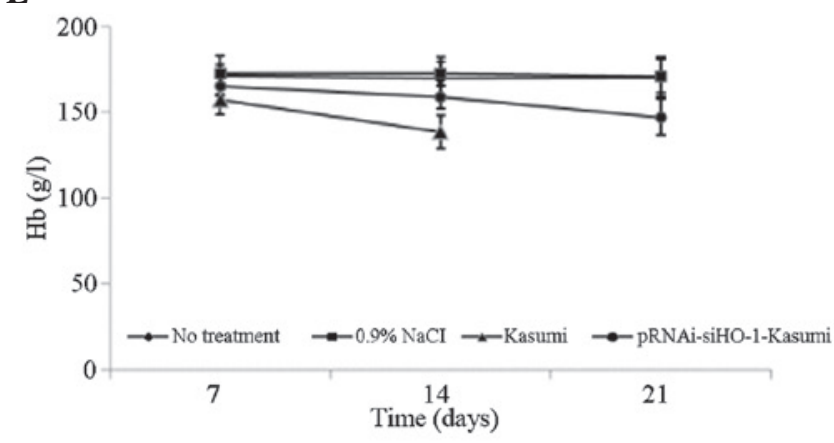

B

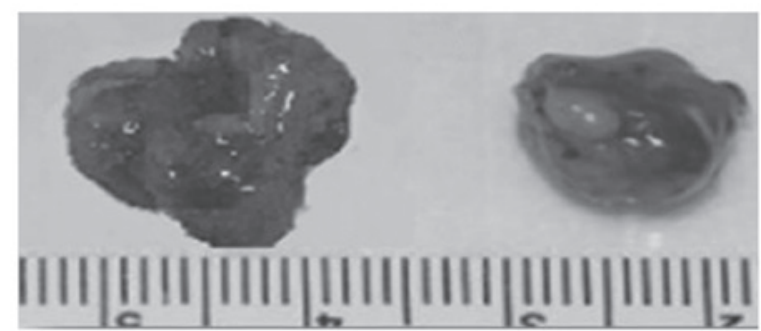

D

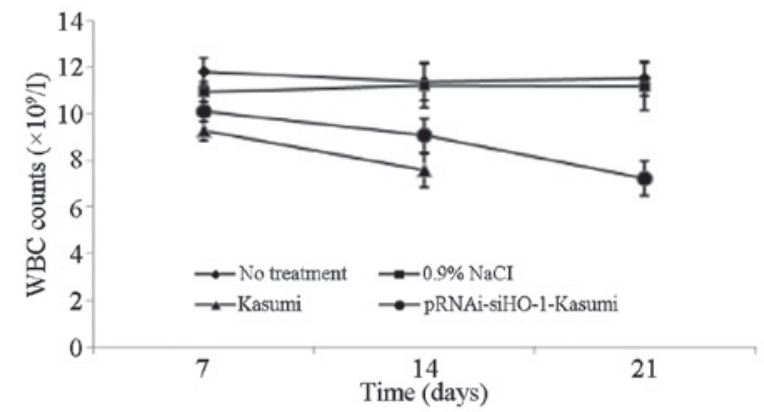

$\mathbf{F}$

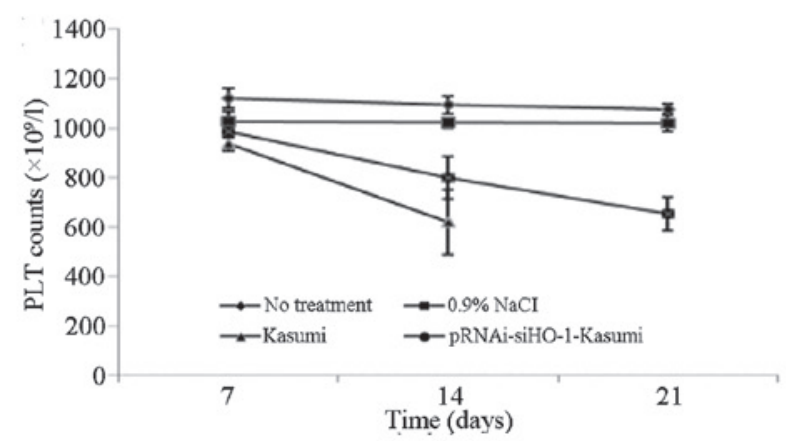

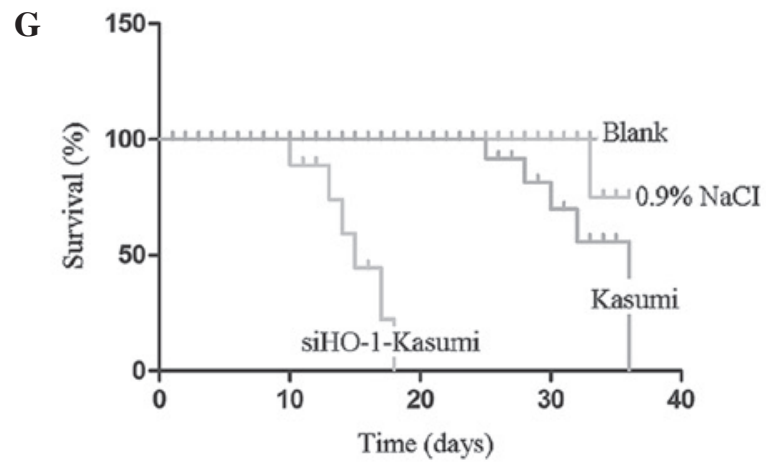

Figure 7. Changes of body weight, leukocyte and platelet counts and hemoglobin levels, and Kaplan-Meier survival curve in the nude mouse model. (A) The Kasumi-1 cell-inoculated nude mouse model; left, Kasumi group; right, pRNAi-siHO-1-K group. (B) Volume of subcutaneous nodules in the nude mouse model; left, Kasumi group; right, pRNAi-siHO-1-K group. (C) Body weight changes of the blank, normal saline, Kasumi and pRNAi-siHO-1-Kasumi groups were recorded every 5 days. The body weights of the Kasumi group and the pRNAi-siHO-1-K group decreased from day 5, and that of the Kasumi group dropped to a greater extent. (D) Changes of leukocyte counts. The count of the Kasumi group reduced significantly ( $\mathrm{P}<0.05)$. (E) Changes of hemoglobin levels. The hemoglobin level in the peripheral blood of the Kasumi group was significantly reduced $(\mathrm{P}<0.05)$. (F) Changes of platelet counts. The count of the Kasumi group was significantly lower than those of the other three groups $(\mathrm{P}<0.05)$. $(\mathrm{G})$ Kaplan-Meier survival curves for evaluating the survival time of nude mice. The pRNAi-siHO-1-K group survived longer than the Kasumi group did. WBC, white blood cell; PLT, platelet; Hb, hemoglobin; HO-1, heme oxygenase-1.

survival time of the Kasumi group, that of the pRNAi-siHO-1-K group was significantly extended $(\mathrm{P}<0.05)$.

Expression of AML1/ETO. In order to assess the degree of infiltration of leukemic cells in different organs, the expression of AML1/ETO in the bone marrow, liver, spleen, lung and kidney was detected. As shown in Fig. 8, the copy numbers $\left(\times 10^{6}\right)$ of AML1/ETO fusion gene in the bone marrow, liver and spleen were $1.036 \pm 0.109,0.230 \pm 0.456$ and $0.160 \pm 0.033$, respectively, in the Kasumi group and $0.333 \pm 0.195,0.084 \pm 0.059$ and $0.058 \pm 0.045$, respectively, in the pRNAi-siHO-1-K group. The copy numbers in the lung and kidney were below the detection limit $\left(1 \times 10^{3}\right)$. The expression level of AML1/ETO was increased in the bone marrow, liver and spleen in the Kasumi and pRNAi-siHO-1-K groups, indicating that the bone marrow, liver and spleen were subjected to diffuse filtration of leukemic cells. By contrast, the expression of AML1/ETO in the lung and kidney was 


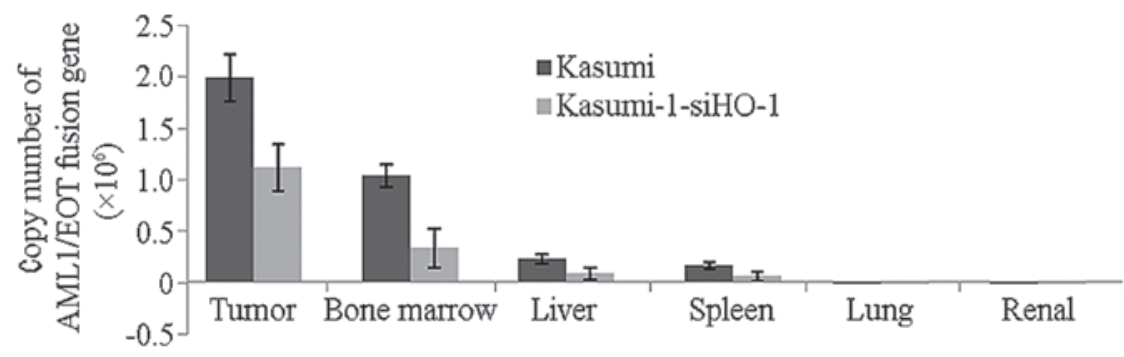

Figure 8. Copy number of AML1/ETO fusion gene as detected by quantitative polymerase chain reaction. AML1/ETO expression in the bone marrow, liver and spleen was significantly higher than that in the lung and kidney. In the Kasumi group, the expression of AML1/ETO was higher than that in the pRNAi-siHO-1-K group. HO-1, heme oxygenase-1.

undetectable, showing that the lungs and kidneys scarcely succumbed to infiltration by leukemic cells. The expression level of AML1/ETO in the Kasumi group was clearly higher than that in the pRNAi-siHO-1-K group.

\section{Discussion}

HO-1 plays a vital role in tumorigenesis (19); HO-1 is generally highly expressed in tumor cells, which affects the response to treatment. Although the functions of HO-1 are tissue-specific, it is a key enzyme that contributes to the onset and development of tumors (20). In addition to heme, drugs as well as physiological and non-physiological stresses, for example, UV radiation, heat, inflammatory factors, microbial toxins and heavy metals, can induce the expression of HO-1 (21-25). The 5'-end of the HO-1 gene contains several binding sites for inflammatory and apoptotic transcription factors such as NF- $\mathrm{BB}, \mathrm{AP}-1$ and Nrf2 (24-26). Therefore, HO-1 expression is involved in cell biological processes through the regulation of transcription factors. Although HO-1 has been verified to exert anti-apoptotic effects on certain solid tumor cells (29), the impact of HO-1 expression in AML cells on biological processes remains to be elucidated.

In the present study, BMMNCs separated from AML-M2 patients were infected with a constructed lentivirus pRNAi-siHO-1-GFP to silence the expression of HO-1. qPCR and western blotting results demonstrated that the level of HO-1 expression in BMMNCs was significantly inhibited.

Subsequently, the effects of targeted HO-1 silencing on the apoptosis of BMMNCs induced by different concentrations of DNR were investigated. The survival rates of BMMNCs after 24, 48 and $72 \mathrm{~h}$ of treatment were detected by the CCK-8 method. The growth inhibition rate increased with rising DNR concentration and with extended treatment time. The group in which HO-1 expression was silenced had higher inhibition rates than the control and the blank vector groups did at each DNR concentration. Following treatment with $12.5 \mu \mathrm{g} / \mathrm{ml}$ DNR, the growth inhibition rates of the three cell groups gradually leveled off. Annexin V-FITC/PI double staining results demonstrated that the apoptotic rate of the pRNAi-siHO-1-BMMNC group was significantly higher than those of the other two groups, suggesting that targeted HO-1 silencing could raise the susceptibility of BMMNCs to DNR by enhancing the pro-apoptotic effects of DNR.

Given that HO-1 silencing inhibited cell survival and promoted apoptosis, the mRNA expression of apoptosis-related genes in the BMMNC and pRNAi-siHO-1-BMMNC groups that had been treated with series concentrations of DNR was detected by qPCR. With rising DNR concentration, the expression levels of caspase-3, -8 and -9 were elevated in a dose-dependent manner. Western blotting results revealed that the protein expression levels of apoptosis-related genes increased following treatment with $5 \mu \mathrm{g} / \mathrm{ml}$ DNR, particularly in the pRNAi-siHO-1-BMMNC group with targeted HO-1 silencing. Thus, targeted HO-1 expression is indicated to be conducive to the apoptosis of BMMNCs by facilitating the activation of these genes. Moreover, HO-1 expression in the pRNAi-siHO-1-BMMNC group was clearly suppressed, but remained dependent upon the concentration of DNR.

The anti-apoptotic and proliferation-inhibiting effects of targeted HO-1 silencing on AML cells were further elucidated in vivo by subcutaneously inoculating nude mice with AML1/ETO-positive Kasumi-1 cells.

The tumor formation outcomes, survival time and body weight changes of the mice were determined to clarify the routine test results of peripheral blood and to observe the infiltration of bone marrow, liver and spleen with grafted tumor cells. No tumors formed in the blank or normal saline groups, while all mice in the Kasumi and pRNAi-siHO-1-K groups developed tumors. They began to bear tumors on days 6 and 10, respectively, and the tumor size of the Kasumi group was significantly larger than that of the pRNAi-siHO-1-K group. Accordingly, HO-1 siRNA may inhibit the malignant proliferation of tumor cells, thus accelerating tumor growth to larger volumes. Kaplan-Meier survival curves demonstrated that the rats of the blank and normal saline groups survived longer, followed by the pRNAi-siHO-1-Kasumi group and the Kasumi group sequentially, indicating that HO-1 expression silencing was able to mitigate the filtration of tumor cells and to prolong the overall survival time. To detect the changes of leukocyte and platelet counts and hemoglobin levels, blood was collected at regular intervals. Since nude mice may die due to excessive bleeding or infections due to frequent sampling, blood samples were collected only on days 7, 14 and 21 after inoculation. Compared with the blank and normal saline groups, the leukocyte and platelet counts and hemoglobin levels of the two experimental groups, particularly those of the Kasumi group, decreased markedly. The results may be ascribed to the suppressed medullary hematopoiesis by the infiltration of Kasumi-1 cells. After blood sampling, the rats of the Kasumi and pRNAi-siHO-1-Kasumi groups did not stop bleeding immediately and required $2 \mathrm{~min}$ 
of local hemostasis, which can be attributed to the reduction of platelet count.

As evidenced by the expression of AML1/ETO, the bone marrow, liver and spleen of the rats of the Kasumi and pRNAi-siHO-1-Kasumi groups were infiltrated with leukemic cells, with those of the former group being more severe. Hence, HO-1 siRNA hindered the invasion of Kasumi-1 cells in vivo.

In short, targeted silencing of HO-1 expression was found to inhibit the proliferation of tumor cells, promote their apoptosis and relieve their infiltration into organs. The findings provide experimental evidence for the gene-targeted therapy of AML-M2. Nevertheless, the regulatory effects of HO-1 silencing on AML treatment in clinical practice require investigation in further studies.

In the present study, the in vivo and in vitro effects of targeted HO-1 silencing on the apoptosis of human M2-type leukemic cells were investigated. HO-1 silencing increased the susceptibility of BMMNCs to DNR and facilitated their apoptosis by caspase activation cascade. HO-1 silencing also suppressed the proliferation of solid tumor cells, enhanced their apoptosis and alleviated infiltration into organs. The results provide valuable evidence for the targeted therapy of M2-type leukemia. However, the detailed mechanisms for the role of downregulated HO-1 expression in the inhibition of tumor cell proliferation and promotion of apoptosis require elucidation by future in-depth studies.

\section{Acknowledgements}

This study was financially supported by the National Natural Science Foundation of China (81270636 and 81070444).

\section{References}

1. Wang YY, Zhou GB, Yin T, et al: AML1-ETO and C-KIT mutation/overexpression in $\mathrm{t}(8 ; 21)$ leukemia: implication in stepwise leukemogenesis and response to Gleevec. Proc Natl Acad Sci USA 102: 1104-1109, 2005.

2. Van Besien K: Allogeneic transplantation for AML and MDS: GVL versus GVHD and disease recurrence. Hematology Am Soc Hematol Educ Program 2013: 56-62, 2013.

3. Yerlikaya A: Expression of heme oxygenase-1 in response to proteasomal inhibition. Protein Pept Lett 19: 1330-1333, 2012.

4. Berberat PO, Dambrauskas Z, Gulbinas A, et al: Inhibition of heme oxygenase-1 increases responsiveness of pancreatic cancer cells to anticancer treatment. Clin Cancer Res 11: 3790-3798, 2005.

5. McAllister SC, Hansen SG, Ruhl RA, et al: Kaposi sarcoma-associated herpesvirus (KSHV) induces heme oxygenase-1 expression and activity in KSHV-infected endothelial cells. Blood 103: 3465-3473, 2004.

6. Was H, Dulak J and Jozkowicz A: Heme oxygenase-1 in tumor biology and therapy. Curr Drug Targets 11: 1551-1570, 2010.

7. Furfaro AL, Piras S, Passalacqua M, et al: HO-1 up-regulation: A key point in high-risk neuroblastoma resistance to bortezomib. Biochim Biophys Acta 1842: 613-622, 2014.

8. Castilho Á, Aveleira CA, Leal EC, et al: Heme oxygenase-1 protects retinal endothelial cells against high glucose-and oxidative/nitrosative stress-induced toxicity. PLoS One 7: e42428, 2012.

9. Zhang L, Liu YL, Chen GX, et al: Heme oxygenase-1 promotes Caco- 2 cell proliferation and migration by targeting CTNND1. Chin Med J (Engl) 26: 3057-3063, 2013.
10. Kongpetch S, Kukongviriyapan V, Prawan A, Senggunprai L, Dukongviriyapan U and Buranrat B: Crucial role of heme oxygenase- 1 on the sensitivity of cholangiocarcinoma cells to chemotherapeutic agents. PLoS One 7: e34994, 2012.

11. Tibullo D, Barbagallo I, Giallongo C, et al: Nuclear translocation of heme oxygenase- 1 confers resistance to imatinib in chronic myeloid leukemia cells. Curr Pharm Des 19: 2765-2770, 2013.

12. Heasman SA, Zaitseva L, Bowles KM, Rushworth SA and Macewan DJ: Protection of acute myeloid leukaemia cells from apoptosis induced by front-line chemotherapeutics is mediated by haem oxygenase-1. Oncotarget 2: 658-668, 2011.

13. Rushworth SA and MacEwan DJ: HO-1 underlies resistance of AML cells to TNF-induced apoptosis. Blood 111: 3793-3801, 2008.

14. Ma D, Fang Q, Li Y, et al: Crucial role of heme oxygenase-1 in the sensitivity of acute myeloid leukemia cell line Kasumi-1 to ursolic acid. Anticancer Drugs 5: 406-414, 2014.

15. Wang JS, Yang C, Fang Q, et al: K562 cell line resistance to nilotinib induced in vitro and preliminary investigation of its mechanisms. Zhonghua Xue Ye Xue Za Zhi 33: 906-910, 2012 (In Chinese).

16. Chen C, Wang JS, Qin D, Yang Y, Yu YY and Fang Q: The effect of retrovirus-mediated HO-1 gene on chronic myeloid leukemia resistance cell K562/A02 apoptosis induced by nilotinib. Zhonghua Xue Ye Xue Za Zhi 33: 383-387, 2012 (In Chinese).

17. Wang JS, Chai BS, Fang Q, He YY, Chen C and Yang C: Effects of HO-1 gene expression on proliferation of imatinib resistant CMl cells. Zhonghua Xue Ye Xue Za Zhi 32: 388-391, 2011 (In Chinese).

18. Gabert J, Beillard E, van der Velden VH, et al: Standardization and quality control studies of 'real-time' quantitative reverse transcriptase polymerase chain reaction of fusion gene transcripts for residual disease detection in leukemia - a Europe Against Cancer program. Leukemia 17: 2318-2357, 2003.

19. Jozkowicz A, Was H and Dulak J: Heme oxygenase-1 in tumors: is it a false friend? Antioxid Redox Signal 9: 2099-2117, 2007.

20. Mayerhofer M, Gleixner KV, Mayerhofer J, et al: Targeting of heat shock protein 32 (Hsp32)/heme oxygenase-1(HO-1) in leukemic cells in chronic myeloid leukemia: a novel approach to overcome resistance against imatinib. Blood 111: 2200-2210, 2008.

21. Pietsch EC, Chan JY, Torti FM and Torti SV: Nrf2 mediates the induction of ferritin $\mathrm{H}$ in response to xenobiotics and cancer chemopreventive dithiolethiones. J Biol Chem 278: 2361-2369, 2003.

22. Ogborne RM, Rushworth SA and O'Connell MA: $\alpha$-Lipoic acid-induced heme oxygenase- 1 expression is mediated by nuclear factor erythroid 2-related factor 2 and p38 mitogen-activated protein kinase in human monocytic cells. Arterioscler Thromb Vasc Biol 25: 2100-2105, 2005.

23. Ogborne RM, Rushworth SA, Charalambos CA and O'Connell MA: Haem oxygenase-1: a target for dietary antioxidants. Biochem Soc Trans 32: 1003-1005, 2004.

24. Liu XM, Peyton KJ, Ensenat D, et al: Nitric oxide stimulates heme oxygenase-1 gene transcription via the Nrf2/ARE complex to promote vascular smooth muscle cell survival. Cardiovasc Res 75: 381-389, 2007.

25. Trekli MC, Riss G, Goralczyk R and Tyrrell RM: Beta-carotene suppresses UVA-induced HO-1 gene expression in cultured FEK4. Free Radic Biol Med 34: 456-464, 2003.

26. Rushworth SA, Bowles KM, Raninga P and MacEwan DJ: NF-kappaB-inhibited acute myeloid leukemia cells are rescued from apoptosis by heme oxygenase-1 induction. Cancer Res 70: 2973-2983, 2010.

27. Wu RP, Hayashi T, Cottam HB, et al: Nrf2 responses and the therapeutic selectivity of electrophilic compounds in chronic lymphocytic leukemia. Proc Natl Acad Sci USA 107: 7479-7484, 2010.

28. Alam J and Cook JL: How many transcription factors does it take to turn on the heme oxygenase-1 gene? Am J Respir Cell Mol Biol 36: 166-174, 2007.

29. Tsai JR, Wang HM, Liu PL, et al: High expression of heme oxygenase-1 is associated with tumor invasiveness and poor clinical outcome in non-small cell lung cancer patients. Cell Oncol (Dordr) 35: 461-471, 2012. 